\title{
THREE-DIMENSIONAL RECORDING OF BASTION MIDDLEBURG MONUMENT USING TERRESTRIAL LASER SCANNER
}

\author{
Z. Majid*, C.L. Lau, A.R. Yusoff \\ Photogrammetry and Laser Scanning Research Group, Faculty of Geoinformation and Real Estate, Universiti Teknologi \\ Malaysia-zulkeplimajid@utm.my, lauchongluh@utm.my, ahmadrazali@utm.my
}

Commission V/2

KEY WORDS: Three-dimensional, Historical Monument, Terrestrial Laser Scanning, Time-off-flight, Point Cloud

\begin{abstract}
:
This paper describes the use of terrestrial laser scanning for the full three-dimensional (3D) recording of historical monument, known as the Bastion Middleburg. The monument is located in Melaka, Malaysia, and was built by the Dutch in 1660. This monument serves as a major hub for the community when conducting commercial activities in estuaries Malacca and the Dutch build this monument as a control tower or fortress. The monument is located on the banks of the Malacca River was built between Stadhuys or better known as the Red House and Mill Quayside. The breakthrough fort on 25 November 2006 was a result of the National Heritage Department through in-depth research on the old map. The recording process begins with the placement of measuring targets at strategic locations around the monument. Spherical target was used in the point cloud data registration. The scanning process is carried out using a laser scanning system known as a terrestrial scanner Leica C10. This monument was scanned at seven scanning stations located surrounding the monument with medium scanning resolution mode. Images of the monument have also been captured using a digital camera that is setup in the scanner. For the purposes of proper registration process, the entire spherical target was scanned separately using a high scanning resolution mode. The point cloud data was pre-processed using Leica Cyclone software. The pre-processing process starting with the registration of seven scan data set through overlapping spherical targets. The post-process involved in the generation of coloured point cloud model of the monument using third-party software. The orthophoto of the monument was also produced. This research shows that the method of laser scanning provides an excellent solution for recording historical monuments with true scale of and texture.
\end{abstract}

\section{INTRODUCTION}

The paper describes the use of terrestrial laser scanning system for the 3D documentation of historical monument known as Bastion Middleburg located in Malacca State, Malaysia. The aim of the research is to evaluate the use of terrestrial laser scanning system known as High Definition Survey Leica C10 for recording and documentation of historical monument. The Leica C10 system is classified as a time-off-flight system that provides the scanning at 360 degree horizontal angle and 270 degree vertical angle with the scanning resolution of 50,000 points per second. The Bastion Middleburg monuments were scanned at seven scanning stations including two scanning stations located on top of the monument. The final output of the scanning job is the $3 \mathrm{D}$ coloured point cloud of the monument.

\section{TERRESTRIAL LASER SCANNING FOR HERITAGE DOCUMENTATION}

A laser scanning 3D measurement system can be divided in three categories which are airborne laser scanning, terrestrial laser scanning and mobile laser scanning. The terrestrial laser scanning system can also categorised as time-off-light based system, phase-based system and triangulation-based system. All the three terrestrial laser scanning system is differed in scanning distance which also known as scanning range, scanning resolution and scanning accuracy. Table 1 shows the comparison between the three types terrestrial laser scanning systems.

\begin{tabular}{|c|c|c|c|}
\hline $\begin{array}{c}\text { Type of } \\
\text { Terrestrial } \\
\text { Laser } \\
\text { Scanning }\end{array}$ & $\begin{array}{c}\text { Scanning } \\
\text { Distance } \\
(\mathrm{m})\end{array}$ & $\begin{array}{c}\text { Scanning } \\
\text { Resolution } \\
\text { (number of } \\
\text { points per } \\
\text { second) }\end{array}$ & $\begin{array}{c}\text { Scanning } \\
\text { Accuracy } \\
(\mathrm{mm})\end{array}$ \\
\hline $\begin{array}{c}\text { Time-Off- } \\
\text { Flight-Based }\end{array}$ & $\begin{array}{c}300 \\
\text { (maximum) }\end{array}$ & 50,000 & $6 \mathrm{~mm}$ \\
\hline Phase-Based & $\begin{array}{c}120 \\
\text { (maximum) }\end{array}$ & 900,000 & $4 \mathrm{~mm}$ \\
\hline $\begin{array}{c}\text { Triangulation- } \\
\text { Based }\end{array}$ & $\begin{array}{c}2.5 \\
\text { (maximum) }\end{array}$ & 300,000 & Micron \\
\hline
\end{tabular}

Table 1. Comparison between the three types of terrestrial laser scanning systems

With the measurement accuracy that can be provided by the terrestrial laser scanning system (as shown above), the system were used in many applications. The main reason why researchers used terrestrial laser scanning system is to record the real geometry and structure of the objects especially in 3D form. In other word, terrestrial laser scanning can provides a successful recording of 3D and complex objects. The time-off-flight and phasebased system were used to record the 3D object such as building, structures like bridge and railway station, while the triangulation-based system were used to capture small 3D object such as human face and historical artefacts. 
Terrestrial laser scanning system has been used to measure and record the geometry and structure of the historical buildings and monuments all over the world. According to Gonzalez J.A et al (2010), terrestrial laser scanning can be used to detect the damage of the historical building. The research involves with the development of the appropriate methodology to study damages on stony materials that constitute historical buildings. The methodology was tested using an intensity data that was derived from the laser scanner data with different technical specifications. The output of the study shows that the intensity data derived from the laser scanning technique potential to be used for the recognition and characterization of certain pathologies in building materials that constitute historical buildings.

According to Entwistle J.A et al (2009), terrestrial laser scanning can be used in the investigation of historical Scottish farming townships through the threedimensional (3D) visualization method. Terrestrial laser scanner can also be used to create photo-realistic virtual copies of landscapes and archaeological features. The study also involves with the development of the methodology for the integration of a high resolution 3D site model with soil chemical data obtained from abandoned settlement site located in the Central Highlands Scotland.

Terrestrial laser scanning is also used to effectively produce accurate and high resolution 3D models of a cave with engravings dating back to the Upper Palaeolithic era (Lerma J.L et al, 2010). For a complete mapping of the complexity cave, terrestrial laser scanning was integrates with close-range photogrammetric method to produce traditional drawings such as sections and elevations, including photo-realistic data in 3D perspective view. The study found that the 3D data acquired from the laser scanner improved archaeological understanding of complex caves and relief panels of prehistoric art with tiny engravings.

According to Zhang Y et al (2015), the terrestrial laser scanning technology have been used for the 3D mapping of historic buildings in China for the purpose of the development of historical database. The terrestrial laser scanning system was used to acquire 3D point cloud data of Shang Su Di, a Ming Dynasty building which is an officially protected heritage site in China. The study also involves a detail mapping of historical building component. The 2D drawing of the component was derived from the $3 \mathrm{D}$ point cloud data using $\mathrm{CAD}$ software.

Calin $M$ et al (2015) used terrestrial laser scanning technology for the purpose of University building conservation for UNESCO recognition. The old university building located in the USAMV Bucharest campus was scanned using terrestrial laser scanning technology to develop a 3D digital model. The 3D information database was also developed to store the 3D model data for assessment and evaluation.

\section{METHODOLOGY}

The methodology implemented in the recording of the Bastion Middleburg monument consists of three phases which are:

a) Phase 1 involves with planning of laser scanning survey

b) Phase 2 involves with data collection

c) Phase 3 involves with data processing

The planning for the laser scanning survey is the very important phase in this research. The planning stage involves with the determination of the scanning targets and the scanning stations. For a very accurate laser scanning survey, the sphere targets were used. The numbers of 14 sphere targets were setup at ten different locations surrounding the monument. For the purpose of accurate registration process, some of the target was located at the higher locations on the monument. The monument was scanned from seven scanning stations including two stations located on top of the monument. Figure 1 shows the location of the sphere targets and the scanning stations.

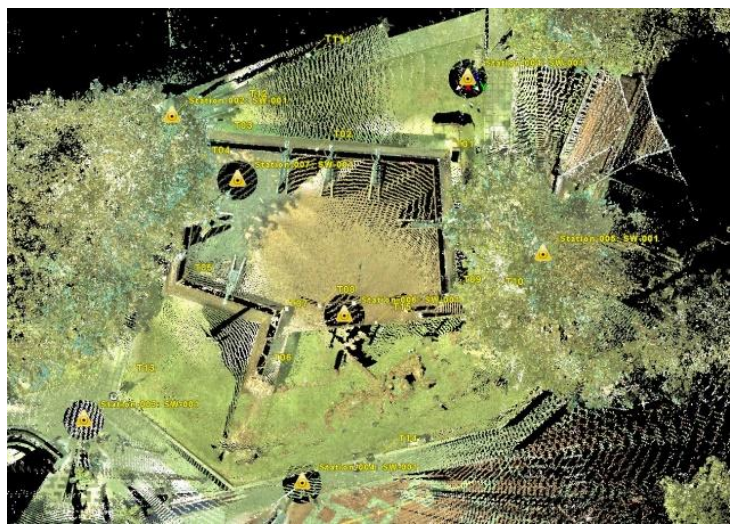

Figure 1. Planning for laser scanning survey - the location of scanning stations and sphere targets

As mentioned earlier, the laser scanning process was carried out using Leica $\mathrm{C} 10$ terrestrial laser scanner. The medium scanning resolution was used in the scanning process. The laser scanning process took 20 minutes per scanning station. The images of the monument were also captured using a built-in digital camera inside the laser scanner. Figure 2 shows the scanning process from one of the scanning station.

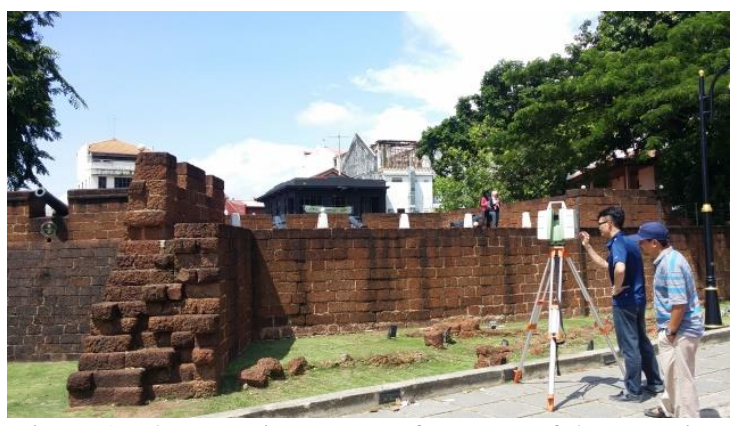

Figure 2. The scanning process from one of the scanning station 
At data processing phase, the research involves with preprocessing and post-processing stages. The preprocessing stage involves with the registration of scanning data and data cleaning. The registration process was carried out using the sphere targets. At least three sphere targets were used to merge two scanning data. The algorithm that was built in the cyclone software measure the centroid coordinates of the sphere target from different view. This capability makes sphere target is the best target to be used in laser scanning survey. The post-processing stage involves with the generation of coloured point cloud data. In the generation of coloured point cloud data, the images taken during the scanning process was mapped on top of the 3D point cloud data of the monument.

\section{RESULTS AND ANALYSIS}

In this research, the output of the scanning is visualized as a coloured point cloud data. Figure 3 shows the coloured point cloud data of the Bastion Middleburg monument.

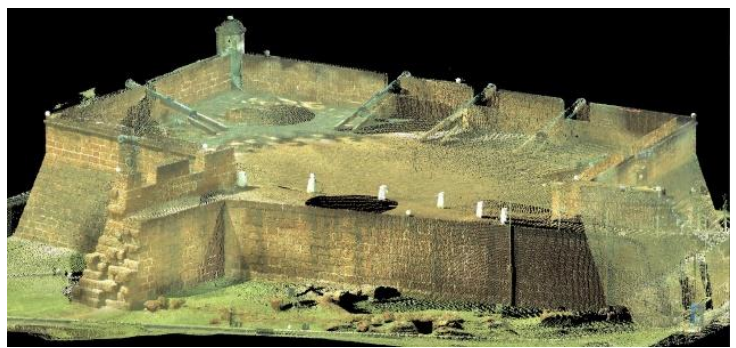

Figure 3. The coloured point cloud data of the Bastion Middleburg monument

The advantage of the laser scanning output is the capability of viewing the 3D coloured point cloud data in many views. Figure 4 shows the viewing of the scanning output from perspective view.

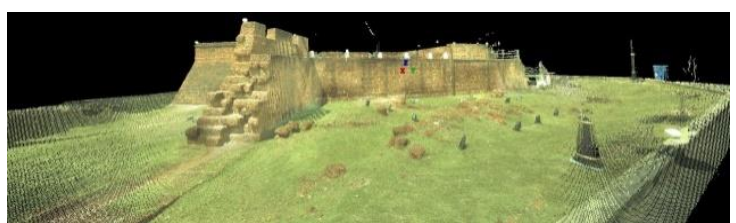

Figure 4. Visualizing of Bastion Middleburg monuments point cloud data from perspective view

Apart from that, the resolution of the scanning data allows the user to get the details of each part of the structure of the monument. Figure 5 and Figure 6 shows the details of the scanning data of the monument.

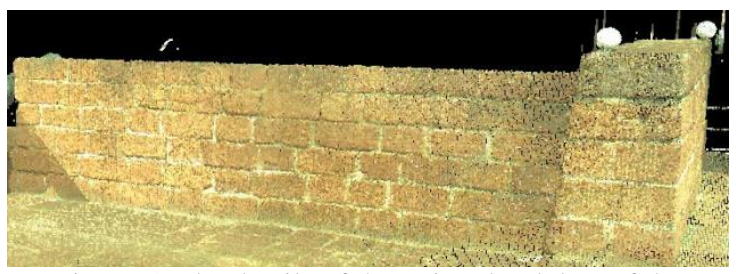

Figure 5. The details of the point cloud data of the monument

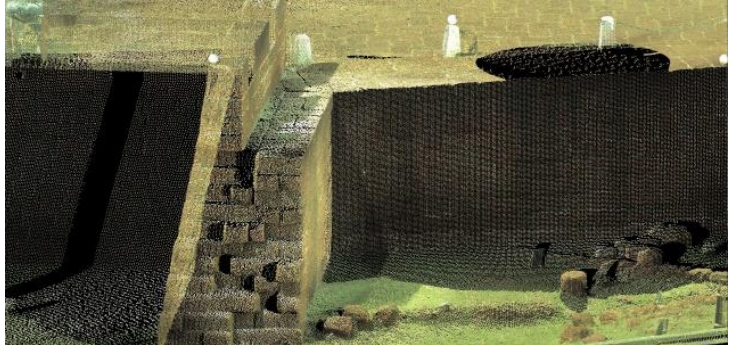

Figure 6. The details of the point cloud data of the front part of the monument

Figure 7 and Figure 8 shows the visualization of the coloured point cloud data from side view and top view of the monument.

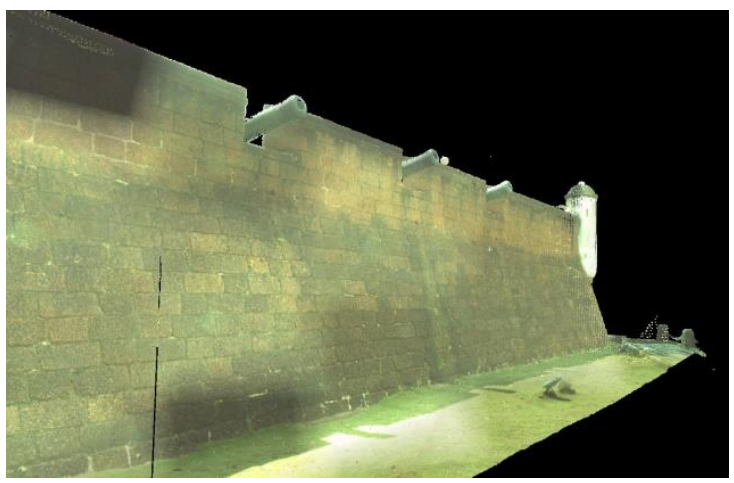

Figure 7. The coloured point cloud data of the side part of the monument

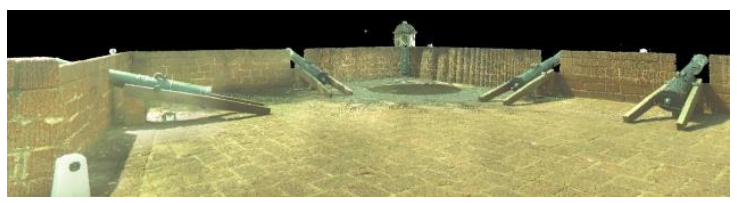

Figure 8 . The coloured point cloud data of the top part of the monument

For the analysis part, the simple comparison analysis was carried out by comparing the laser scanning coloured point cloud data with the image of the real object (monument). The image of the real object was acquired using high resolution digital camera. Figure 9 shows the simple comparison analysis result.
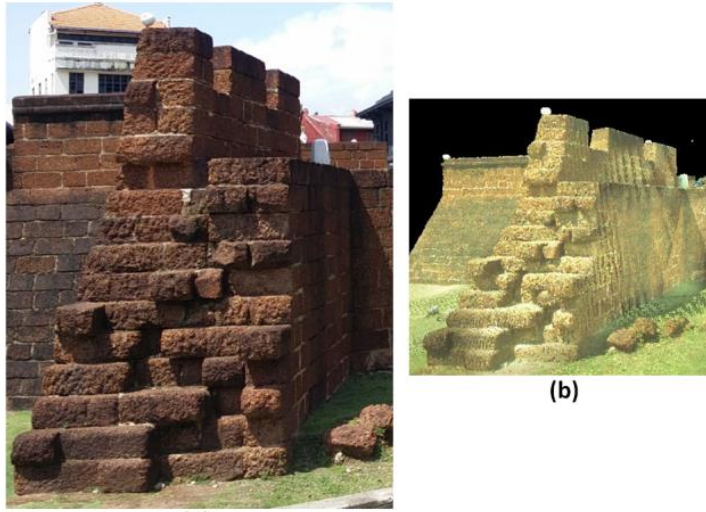

(b)

Figure 9. Simple comparison analysis between the real object (a), and the coloured point cloud data (b) 
Figure 9 show that the laser scanning method delivers the similar geometry and structure of the monument. From the visualization aspect, the similarity between the laser scanning data and the real object can be considered as a perfect match. The problem with the coloured point cloud data is the tone of the texture. This problem may happen because of the resolution of the built in camera inside the laser scanner. This problem can be solved by combining the laser scanning data with the images acquire from external digital camera with high resolution capabilities.

\section{CONCLUSION}

The paper describes the experience of using terrestrial laser scanning technology for the 3D documentation of historical monument known as the Bastion Middleburg. The number of seven scanning stations and fourteen sphere targets was used in the scanning survey. The final output from the laser scanning task is the $3 \mathrm{D}$ coloured point cloud of the monument with true scale and texture. As conclusion, the terrestrial laser scanning technology has proven to be the best data acquisition technique for recording of historical monument.

\section{ACKNOWLEDGEMENT}

The output from this research will be used as a benchmark for the UAV LiDAR survey using Phoenix AL3 System that was fully funded by the Ministry of Higher Education Malaysia under research vot 4L149.

\section{REFERENCES}

Calin M, Erghelegiu B, Manea R, Virsta A and Salagean $T$ (2015), University Building Conservation Using Terrestrial Laser Scanning Technique, Buletin UASVM Horticulture 72(1)/2015 (ISSN 1843-5254).
Entwistle J.A, McCaffrey K.J.W and Abrahams P.W (2009), Three-dimensional (3D) Visualization: The Application of Terrestrial Laser Scanning in the Investigation of Historic Scottish Farming Townships, Journal of Archaeological Science, Volume 36, Issue 3, March 2009, pages 860-866.

Gonzalez J.A, Rodriguez B.R, Aguilera D.G and Brea M.T.R (2010), Terrestrial Laser Scanning Intensity Data Applied to Damage Detection for Historical Buildings, Journal of Archaeological Science, Volume 37, Issue 12, December 2010, pages 3037-3047.

Lerma J.L, Navarro S, Cabrelles $M$ and Villaverde V (2010), Terrestrial Laser Scanning and Close-Range Photogrammetry for 3D Archaeological Documentation: The Upper Palaeolithic Cave of Parpallo As A Case Study.

Zhang $\mathrm{Y}$, Zhang $\mathrm{Y}$, Shen $\mathrm{Z}$, Nishino $\mathrm{T}$ and Chen $\mathrm{X}$ (2015), 3D Laser Scanning Technology-based Historic Building Mapping for Historic Preservation, International Review For Spatial Planning and Sustainable Development, Vol. 3, No. 2 (2015), 53-67. 